\title{
THE POLYNOMIAL APPROXIMATION PROPERTY IN FOCK-TYPE SPACES
}

\author{
A. A. BORICHEV*
}

\section{Introduction.}

Let $\lambda(r)$ be a positive function on $[0, \infty), \log r=o(\lambda(r)), r \rightarrow \infty$. Then the Fock space $F_{\lambda}$, associated with $\lambda$, consists of all entire functions $f$ such that

$$
\|f\|_{F_{\lambda}}^{2}=\int_{\mathrm{C}}|f(z)|^{2} e^{-\lambda(|z|)} d m_{2}(z)<\infty
$$

where $d m_{2}$ is Lebesgue area measure. We deal with the following question, posed and discussed in [9] (and earlier in [8]).

Do the polynomial multiples of an entire function $\phi$ span (in $F_{\lambda}, \lambda(r)=r^{2}$ ) all functions which vanish wherever $\phi$ vanishes? We take into account the multiplicities of zeros, and require that all the polynomial (or even exponential polynomial) multiples of $\phi$ belong to $F_{\lambda}$. In other words, the question is whether the polynomials are dense in the Hilbert space $F_{\lambda, \phi}$ of analytic (entire) functions square summable with respect to the measure $|\phi(z)|^{2} e^{-\lambda(|z|)} d m_{2}(z)$. Let us remark here that the general problem of weighted polynomial approximation for weights vanishing at some inner points of the domain of analyticity is not yet sufficiently investigated.

For some classes of $\lambda$ we construct $\phi$ for which the answer to our question is negative.

The method used is close to that in [2,3]. The idea is to produce an element $\phi$ of $F_{\lambda}$ having extremal growth along a sufficiently massive subset of the plane. Then a Phragmén-Lindelöf type argument shows that if polynomial multiples $p_{n} \phi$ converge to an element in $F_{\lambda}$, then the polynomials $p_{n}$ cannot be large enough to approximate all the elements in $F_{\lambda, \phi}$. To use this

\footnotetext{
* The research was supported in part by the Swedish Natural Science Research Council. Received February 10, 1996.
} 
argument we need first to consider (in Section 5) a problem on the harmonic measure in the unit disc. In Section 2 we formulate the main results. An element of extremal growth is constructed in Section 4. Our construction uses an auxiliary lemma describing general weight functions $\lambda$ as restrictions on the positive axis of entire functions with positive coefficients, which is proved in Section 3. Finally, the Phragmén-Lindelöf type argument is given in Section 6.

The author is thankful to H. S. Shapiro for calling his attention to this problem.

\section{Main results.}

We are going to deal with the following two conditions on a strictly increasing $C^{2}$-smooth function $\lambda$.

(i) $\lambda^{\prime}(2 t) \leq c \lambda^{\prime}(t)$ for some $c$ and sufficiently big $t$.

(ii $\left.{ }_{k}\right) \liminf \inf _{t \rightarrow \infty} \exp (-k t)(\lambda(\exp t))^{\prime \prime}>0$.

Note that condition ( $\left.\mathrm{ii}_{1}\right)$ always holds for convex $\lambda$, and condition ( $\left.\mathrm{ii}_{k}\right)$ holds for $\lambda(x)=x^{s}, s \geq k$.

For $0<p<\infty$ consider the family of Fock-type spaces

$F_{\lambda}^{p}=$ the set of all entire functions $f$ such that $\int_{C}|f(z)|^{p} e^{-\lambda(|z|)} d m_{2}(z)<\infty$.

They are Banach spaces for $1 \leq p<\infty$, and complete metric spaces for $0<p<1$.

THEOREM. Let $\lambda$ satisfy conditions $(i)$ and $\left(i_{k}\right)$ for some integer number $k$, $k \geq 1,0<p<\infty$, and let $s$ be a positive number such that $s<k / 2$. There exist an entire function $f$ and a positive number a such that $e^{-a z^{k}} f \in F_{\lambda}^{p}$,

$$
\int_{\mathrm{C}}|f(z)|^{p} e^{|z|^{s}} e^{-\lambda(|z|)} d m_{2}(z)<\infty
$$

but

$$
e^{-a z^{k}} f \notin \underset{F_{\lambda}^{p}}{\operatorname{clos}} \mathscr{L}\left(\left\{z^{n} f, n \geq 0\right\}\right) .
$$

Here $\mathscr{L}(E)$ is the linear hull of $E$.

COROllary 1. Let $\lambda$ satisfy conditions (i) and (ii $\left.{ }_{1}\right), 0<p<\infty$. There exist an entire function $f$ and a positive number a such that $e^{-a z} f \in F_{\lambda}^{p}$, and for every $n \geq 0, z^{n} f \in F_{\lambda}^{p}$, but

$$
e^{-a z} f \notin \operatorname{clos}_{F_{\lambda}^{p}} \mathscr{L}\left(\left\{z^{n} f, n \geq 0\right\}\right) .
$$


COROllary 2. Let $\lambda$ satisfy conditions (i) and ( $\left.\mathrm{ii}_{3}\right), 0<p<\infty$. There exist an entire function $f$ and a positive number a such that $e^{-a z^{3}} f \in F_{\lambda}^{p}$, and for every $b \in \mathrm{C}, e^{b z} f \in F_{\lambda}^{p}$, but

$$
e^{-a z^{3}} f \notin \underset{F_{\lambda}^{p}}{\operatorname{clos}} \mathscr{L}\left(\left\{z^{n} f, n \geq 0\right\}\right) .
$$

REMARK. Actually, the proof of Theorem shows that for $k \geq 3$ there exist such $f$ that the function $e^{-a z^{k}} f$ cannot be approximated in $F_{\lambda}^{p}$ even by exponential polynomial multiples of $f$ or, in general, by products $h^{v} f$ where $h^{v}$ are entire functions of order less than or equal to $v, v<k / 2$.

REMARK. The (unbounded) operator $T_{f}$ of multiplication by $f$ and more general Toeplitz operators on the Fock space, $p=2, \lambda(t)=t^{2}$, were studied by J. Janas in a series of recent papers. J. Janas and J. Stochel use in [6] the notion of a core for an unbounded operator $T$. This is just a linear subspace $L$ of the domain $D(T)$ of $T$ such that the graph of $T$ is the closure of the graph of $T \mid L$.

H. S. Shapiro pointed out that entire functions $f$ constructed in Theorem (for $p=2,0<s<1, \lambda(t)=t^{2}$ ) satisfy the following property:

Polynomials belong to $D\left(T_{f}\right)$, but the set of polynomials is not a core for $T_{f}$.

\section{Auxiliary lemma.}

To prove Theorem we use an auxiliary statement. It is similar to results on Legendre-type transforms in [10], [4], [1, Appendix]. In fact, to prove Theorem itself it would be enough to use only the result from [4]. However, our lemma which gives a criterion for a weight function to be representable up to a multiplicative constant by an entire function with positive coefficients seems to be of independent interest. The proof is close to that in [4].

Lemma 1. If $\sigma$ is $C^{2}$-smooth function on $\mathrm{R}_{+}$such that $\sigma^{\prime}(0)=1$,

$$
\sigma^{\prime \prime}(x) \geq \alpha>0, \quad x \geq 0,
$$

then there exists an entire function $g$,

$$
g(z)=\sum_{n \geq 0} x_{n} z^{n}, \quad x_{n} \geq 0
$$

such that

$$
0<c_{1} \leq g(x) e^{-\sigma(\log x)} \leq c_{2}, \quad x \geq 1,
$$

where $c_{1}$ and $c_{2}$ depend only on $\alpha$.

Proof. Let points $a_{n} \in \mathrm{R}_{+}$be defined by the equalities 


$$
\sigma^{\prime}\left(a_{n}\right)=n
$$

and put

$$
b_{n}=\sigma\left(a_{n}\right) .
$$

For every $m, n \in \mathrm{N}, m<n$, denote

$$
\begin{gathered}
I_{m, n}=\left[a_{m}, a_{n}\right], \\
\delta_{m, n}=\max _{t \in I_{m, n}} \min \left\{\sigma(t)-b_{m}-m\left(t-a_{m}\right), \sigma(t)-b_{n}-n\left(t-a_{n}\right)\right\}, \\
\gamma_{m, n}=\min \left\{b_{n}-b_{m}-m\left(a_{n}-a_{m}\right), b_{m}-b_{n}-n\left(a_{m}-a_{n}\right)\right\} .
\end{gathered}
$$

It is clear that $\gamma_{m, n} \geq \delta_{m, n}$. Condition (1) implies that

$$
0<a_{n+1}-a_{n}<c
$$

for some $c$ depending only on $\alpha$. Choose a sequence $\left\{n_{k}\right\}_{k \geq 1}$ in the following way. Put $n_{1}=1$. If $n_{k}$ is already chosen, take as $n_{k+1}$ the minimal integer number $s, s \geq n_{k}+1$, such that $\delta_{n_{k}, s} \geq 1$. Then

$$
\gamma_{n_{k}, n_{k+1}} \geq \delta_{n_{k}, n_{k+1}} \geq 1
$$

It is clear that $a_{n_{k+1}}-a_{n_{k}}<c$ for some $c$ depending only on $\alpha$.

Let us verify that for some positive constants $c_{1}$ and $c_{2}$ depending only on $\alpha$,

$$
c_{1} \leq \exp (-\sigma(t)) \sum_{k \geq 1} \exp \left[b_{n_{k}}+n_{k}\left(t-a_{n_{k}}\right)\right] \leq c_{2}, \quad t \geq 0 .
$$

Indeed, the left estimate follows from the inequality

$$
\delta_{n_{k}, n_{k+1}}<c
$$

where $c$ depends only on $\alpha$, which is an immediate consequence of convexity of $\sigma$ and the inequalities

$$
\begin{aligned}
& \delta_{n_{k}, n_{k+1}-1}<1, \\
& a_{n_{k+1}}-a_{n_{k}}<c .
\end{aligned}
$$

The right estimate is a consequence of the inequality

$$
\gamma_{n_{k}, n_{m}} \geq|k-m|
$$

which follows from (2) by induction. 
Thus, we can put

$$
g(z)=\sum_{k \geq 1} e^{\left(b_{n_{k}}-n_{k} a_{n_{k}}\right)} z^{n_{k}}
$$

and the lemma is proved.

\section{The proof of Theorem. Construction of an extremal function.}

For the sake of simplicity we consider only the case $p=1$. For other $p$ the argument is identical. In this Section we construct $f$ and verify its growth properties. In the next two sections we prove that $e^{a z^{k}} f$ cannot be approximated by polynomial multiples of $f$.

Pick $\theta, b$ such that $s<b<\theta<k / 2$. Condition ( ii $_{k}$ ) on $\lambda$ implies that for some $a>0$, we can redefine the function $\sigma$,

$$
\sigma(t)=\lambda\left(e^{t / k}\right)-a e^{t}-e^{b t / k},
$$

on a finite interval of $R_{+}$in such a way that it satisfies condition (1). Lemma 1 gives us an entire function $g$ with positive Taylor coefficients such that

$$
0<c_{1} \leq g(x) \exp \left(-\lambda\left(x^{1 / k}\right)+a x+x^{b / k}\right) \leq c_{2}<\infty, \quad x \geq 0 .
$$

Put $f(z)=g\left(z^{k}\right) e^{a z^{k}}$. Then

$\int_{\mathrm{C}}|f(z)| e^{|z|^{s}} e^{-\lambda(|z|)} d m_{2}(z) \leq C \int_{0}^{\infty} \exp \left(\lambda(r)-a r^{k}-r^{b}+a r^{k}+r^{s}-\lambda(r)\right) r d r<\infty$.

Analogously, $g=e^{-a z^{k}} f \in F_{\lambda}^{1}$.

\section{Harmonic measure in the unit disc.}

To complete the proof we need first to consider the following problem. Let $0<\delta<1 / 2, k \geq 1$,

$$
\begin{array}{r}
\Omega_{k}=\mathrm{D} \backslash \bigcup_{n=0}^{k-1}[\delta, 1) e^{2 \pi i n / k}, \\
\alpha_{k}=\max _{|z|=\delta} \omega\left(\Omega_{k}, \mathrm{~T}, z\right),
\end{array}
$$

where $\omega(\cdot, \cdot, \cdot)$ is the harmonic measure. What is the asymptotics of $a_{k}$ for $k \rightarrow \infty$ ?

Lemma 2. For an absolute constant $\beta$,

$$
a_{k}<\beta \delta^{k / 2} \text {. }
$$


Proof. We just apply two conformal mappings: $z \rightarrow z^{k}$ and $z \rightarrow\left(\delta^{k}-z\right)^{1 / 2}$ where the branch of the square root function is taken with cut along $(-\infty, 0)$. If $\Omega_{k}^{\prime}$ is the set of the points $z$ in the right half-plane such that $\delta^{k}-z^{2} \in \mathrm{D}$, and $\Gamma_{k}$ is the subset of $\Omega_{k}^{\prime}$ consisting of the points $z$ such that $\delta^{k}-z^{2} \in \delta^{k} \mathrm{~T}$, then

$$
\max _{|z|=\delta} \omega\left(\Omega_{k}, \mathrm{~T}, z\right)=\max _{z \in \Gamma_{k}} \omega\left(\Omega_{k}^{\prime}, \Omega_{k}^{\prime} \cap i \mathrm{R}, z\right) .
$$

The last maximum is easily estimated as const $\cdot \delta^{\mathrm{k} / 2}$.

Now fix $k$. Let $\varepsilon$ be a small positive number, $D \geq 2$, and let $E_{n}$, $0 \leq n \leq k-1$, be open subsets of angles

$$
\left\{z=r e^{i \alpha}:\left|\alpha-\frac{2 \pi n}{k}\right|<\varepsilon\right\}
$$

such that their radial projections $E_{n}^{\prime}$ satisfy the condition

$$
m\left([1 / D, 1) \backslash E_{n}^{\prime}\right)<\varepsilon,
$$

where $m$ is Lebesgue measure on $\mathbf{R}$.

Hall's lemma (see [5, p. 367]) and the theorem on two constants (see [7, p. 257]) permit us to draw the following corollary of Lemma 2.

Lemma 3. For every $\theta, 0<\theta<k / 2$, there exist such small positive number $\varepsilon, \varepsilon=\varepsilon(\theta, k)$, and big integer number $D, D=D(\theta, k)$, that for every function $h$ subharmonic in $\mathrm{D}$ and such that

$$
\begin{aligned}
& h(z) \leq c_{1}, \quad z \in \mathrm{D}, \\
& h(z) \leq c_{2}, \quad z \in E_{n}, \quad n=0, \ldots, k-1,
\end{aligned}
$$

where $E_{n}$ are as above, we have

$$
h(z) \leq c_{1} D^{-\theta}+c_{2}\left(1-D^{-\theta}\right), \quad|z|=1 / D .
$$

\section{The end of the proof. A Phragmén-Lindelöf type argument.}

Now, suppose that

$$
p_{k} f \stackrel{F_{\lambda}^{1}}{\longrightarrow} g
$$

for a sequence of polynomials $\left\{p_{k}\right\}_{k \geq 1}$. Then for some $c$ independent of $k$ we have

$$
\int_{\mathrm{C}}\left|\frac{p_{k}(z)}{c}\right| f(z) \mid e^{-\lambda(|z|)} d m_{2}(z) \leq 1 .
$$


We are going to verify that (4) implies an estimate (uniform in $k$ ) on the polynomials $p_{k}$ which is incompatible with pointwise convergence of $p_{k}$ to $e^{-a z^{k}}$.

Take a polynomial $p$ such that

$$
\int_{\mathrm{C}}|p(z)||f(z)| e^{-\lambda(|z|)} d m_{2}(z) \leq 1 .
$$

Fix $\varepsilon=\varepsilon(\theta, k), D=D(\theta, k)$ from Lemma $3(\theta$ is chosen in Section 4). Put $\gamma(t)=\sigma(k \log t)+a t^{k}=\lambda(t)-t^{b}$. Furthermore, take $m \geq 1$, and let $N$ be the minimal integer number bigger than $\lambda^{\prime}\left(D^{m}\right)$.

Consider the rectangle $A_{m}$,

$$
A_{m}=\left\{z \in \mathrm{C}: D^{m} \leq \operatorname{Re} z \leq D^{m+1}, N|\operatorname{Im} z| \leq 1\right\},
$$

and its subset $E_{m}$,

$$
E_{m}=\left\{z \in A_{m}:|p(z)|<N \exp \left(D^{b(m+2)}\right)\right\} .
$$

$A_{m}$ is the union of $\left(D^{m+1}-D^{m}\right) N / 2$ squares

$$
\begin{gathered}
A_{m, s}=\left\{z \in \mathrm{C}: D^{m}+2(s-1) / N \leq \operatorname{Re} z \leq D^{m}+2 s / N, N|\operatorname{Im} z| \leq 1\right\}, \\
1 \leq s \leq\left(D^{m+1}-D^{m}\right) N / 2 .
\end{gathered}
$$

By conditions (i) on $\lambda$ and (1) on $\sigma$,

$$
\begin{gathered}
\max \left\{\gamma^{\prime}(x): D^{m-1} \leq x \leq D^{m+1}\right\} \leq C_{1} N \\
\left|\gamma(\log |z|)-\gamma\left(\log \left(D^{m}+(2 s-1) / N\right)\right)\right| \leq C_{2}, \quad z \in A_{m, s}, \\
1 \leq s \leq\left(D^{m+1}-D^{m}\right) N / 2
\end{gathered}
$$

where $C_{1}, C_{2}$ do not depend on $m$ and $s$. Furthermore, by (3),

$$
\begin{array}{ll}
\log |f(z)| \leq \gamma(|z|)+C_{3}, & z \in \mathrm{C}, \\
\log |f(x)| \geq \gamma(x)+C_{4}, & x \geq 0 .
\end{array}
$$

Since the function $\log |f|$ is subharmonic, we obtain that

$$
\log |f(z)| \geq \gamma(|z|)+C_{5}
$$

on a subset $B_{m, s}$ of $A_{m, s}$ whose Lebesgue measure $m_{2}\left(B_{m, s}\right)$ is at least $(1-\varepsilon / 2) m_{2}\left(A_{m, s}\right)$, for some constants $C_{3}, C_{4}, C_{5}$ independent of $m$ and $s$. Denote $B_{m}=\cup_{s} B_{m, s}$. We have 


$$
\begin{gathered}
1 \geq \int_{B_{m} \backslash E_{m}}|p(z)||f(z)| e^{-\lambda(|z|)} d m_{2}(z) \geq \\
\geq C \int_{B_{m} \backslash E_{m}} N \exp \left(D^{b(m+2)}\right) e^{\gamma(|z|)} e^{-\lambda(|z|)} d m_{2}(z)= \\
=C N \exp \left(D^{b(m+2)}\right) \int_{B_{m} \backslash E_{m}} e^{-|z|^{b}} d m_{2}(z) .
\end{gathered}
$$

Therefore, for sufficiently big $m, m_{2}\left(B_{m} \backslash E_{m}\right)<\varepsilon m_{2}\left(B_{m}\right) / 2$, and as a result, the length of the radial projection of $E_{m}$ on $\mathrm{R}$ is bigger than $(1-\varepsilon)\left(D^{m+1}-D^{m}\right)$.

The same argument works for the rectangles $A_{m}^{n}$,

$$
A_{m}^{n}=e^{2 \pi i n / k} A_{m}, \quad 0 \leq n \leq k-1,
$$

and their subsets $E_{m}^{n}$,

$$
E_{m}^{n}=\left\{z \in A_{m}^{n}:|p(z)|<N \exp \left(D^{b(m+2)}\right)\right\} .
$$

Now we use the following Phragmén-Lindelöf type reasoning. Since $p$ is a polynomial, there exists $C_{p}$ such that

$$
\log |p(z)| \leq C_{p} \log (1+|z|) .
$$

Fix sufficiently big $m_{0}$, and take some $m, m \geq m_{0}$. Then the function $h(z)=\log |p(z)|$ satisfies the following estimates:

$$
\begin{aligned}
& h(z) \leq c C_{p} m, \quad|z|=D^{m+1}, \\
& h(z) \leq c D^{b m}+\log \left(\lambda^{\prime}\left(D^{m}\right)\right), \quad z \in E_{m}^{n}, 0 \leq n \leq k-1,
\end{aligned}
$$

for some $c$ which does not depend on $p$. Thus, Lemma 3 applies to the function $h\left(D^{m+1} z\right)$ subharmonic in $\mathrm{D}$ and shows that

$$
h(z) \leq c C_{p} m D^{-\theta}+\left[c D^{b m}+\log \left(\lambda^{\prime}\left(D^{m}\right)\right)\right], \quad|z|=D^{m} .
$$

Repeating this procedure $\left(m-m_{0}\right)$ more times we obtain that

$h(z) \leq c C_{p} m D^{-\theta\left(m-m_{0}+1\right)}+\sum_{j=0}^{m-m_{0}} D^{-\theta j}\left[c D^{b\left(m_{0}+j\right)}+\log \left(\lambda^{\prime}\left(D^{m_{0}+j}\right)\right)\right], \quad|z|=D^{m_{0}}$.

When $m$ tends to $\infty$, we get

$$
h(z) \leq \sum_{j=0}^{\infty} D^{-\theta j}\left[c D^{b\left(m_{0}+j\right)}+\log \left(\lambda^{\prime}\left(D^{m_{0}+j}\right)\right)\right], \quad|z|=D^{m_{0}} .
$$

Condition (i) on $\lambda$ and the inequality $b<\theta$ give us now that 


$$
h(z) \leq c_{1} D^{b m_{0}}, \quad|z|=D^{m_{0}},
$$

and by the maximum principle,

$$
|p(z)| \leq e^{c_{2}\left(|z|^{b}+1\right)}, \quad z \in \mathrm{C},
$$

where $c_{1}$ and $c_{2}$ do not depend on $p$.

Therefore, if polynomials $p_{k}$ satisfy estimate (4), they cannot approximate the function $\exp \left(-a z^{k}\right)$. Theorem is proved.

\section{REFERENCES}

1. A. A. Borichev, H. Hedenmalm, Completeness of translates in weighted spaces on the halfline, Acta Math. 174 (1995), 1-84.

2. A. A. Borichev, H. Hedenmalm, Cyclicity in Bergman-type spaces, International Mathematics Research Notices no.5 1995, 253-262.

3. A. A. Borichev, H. Hedenmalm, Cyclicity in Bergman-type spaces and harmonic functions of maximal growth, Uppsala University Department of Mathematics Preprints 1994: 47, $25 \mathrm{pp}$.

4. Y. Domar, Closed primary ideals in a class of Banach algebras, Math. Scand. 7 (1959), 109125.

5. J. Garnett, Bounded Analytic Functions, Academic Press, 1981.

6. J. Janas, J. Stochel, Unbounded Toeplitz operators in the Segal-Bargmann space, II, J. Funct. Anal. 126 (1994), 418-447.

7. P. Koosis, The Logarithmic Integral, vol. I, Cambridge University Press, Cambridge, 1988.

8. D. J. Newman, H. S. Shapiro, Fisher spaces of entire functions, Proc. Sympos. Pure Math. 11 (1968), 360-369.

9. H. S. Shapiro, Comparative approximation in two topologies, Approximation Theory, Banach Center Publ., Warsaw 4 (1979), 225-232.

10. V. Vidensky, On normally increasing functions, Uspekhi Mat. Nauk 9 no. 2 (1954), 212-213 [in Russian].

DEPARTMENT OF MATHEMATICS

UPPSALA UNIVERSITY

SWEDEN

\author{
CURRENT ADDRESS \\ DEPARTMENT OF MATHEMATICS \\ UNIVERSITY OF BORDEAUX I \\ 351, COURS DE LA LIBERATION \\ 33405 TALENCE, FRANCE \\ E-mail: borichev@math.u-bordeaux.fr
}

